Synchrotron Radiation Studies on Even-Odd and Odd-Even Nylons

This article has been downloaded from IOPscience. Please scroll down to see the full text article.

2010 IOP Conf. Ser.: Mater. Sci. Eng. 14012006

(http://iopscience.iop.org/1757-899X/14/1/012006)

View the table of contents for this issue, or go to the journal homepage for more

Download details:

IP Address: 147.83.141.22

The article was downloaded on 09/12/2010 at 14:11

Please note that terms and conditions apply. 


\title{
Synchrotron Radiation Studies on Even-Odd and Odd-Even Nylons
}

\author{
A. Ricart, D. Soto, L. Franco, L. T. Morales, J. Puiggalí ${ }^{1}$ \\ Departament d'Enginyeria Química, Universitat Politècnica de Catalunya, Av. \\ Diagonal 647, E-08028, Barcelona, SPAIN \\ E-mail: Jordi.Puiggali@upc.es
}

\begin{abstract}
Aliphatic polyamides derived from odd diamine or odd dicarboxylic acid units cannot adopt a conventional sheet structure when molecular chains have an all trans conformation. However, typical fiber diffraction patterns of this sheet structure were observed in several polyamides derived from odd units such as nylons 65 and 56. Consequently, a new structure based on the establishment of intermolecular hydrogen bonds along two different directions was postulated. Real-time temperature dependence of X-ray diffraction patterns for nylons 65 and 56 was studied by synchrotron radiation to gain understanding of the Brill transition usually occurring in polyamides. Significant differences were found between the temperature dependence of both nylons, although a transition towards a monoclinic structure characterized by a single equatorial reflection around $0.425-0.420 \mathrm{~nm}$ was observed to occur at high temperatures. This transition was reversible for nylon 65 only. In this case, a characteristic hysteresis effect was found.
\end{abstract}

\section{Introduction}

The structure of aliphatic polyamides is usually based on the stacking of sheets composed of hydrogen-bonded molecular chains with a planar zig-zag conformation when the repeat unit has an even number of carbon atoms in the main chain [1] (e.g. nylons 6 [2] and 66 [3]). X-ray fiber diffraction patterns of such a structure are characterized by two strong equatorial reflections that appear at spacings close to 0.44 and $0.37 \mathrm{~nm}$. These reflections are associated with interchain distances within and between sheets, respectively.

Sheet structures are energetically favored when $\mathrm{NH}$ and $\mathrm{CO}$ groups of neighboring chains face each other and all possible hydrogen bonds with an appropriate geometry form (e.g. angles close to $180^{\circ}$ for $\mathrm{N}-\mathrm{H} . . . \mathrm{O}$ and $\mathrm{H} . . . \mathrm{OC}$ interactions). These optimal intermolecular interactions cannot be obtained when polyamides are constituted by diamines and/or dicarboxylic acids with an odd number of carbon atoms and an all trans molecular conformation (Figure 1a). In this case, a pseudohexagonal structure characterized by a single strong reflection near $0.420-0.415 \mathrm{~nm}$ seems to be favored (e.g. the $\gamma$ form postulated for nylon 77 [4]). However, it has recently been reported that several polyamides derived from odd diamines (e.g. nylons 56 [5], 92 [6] and 5-10 [7]) or odd dicarboxylic acids (e.g. nylons 65 [8] and 69 [9]) have room temperature structures characterized by two strong equatorial reflections near 0.43 and $0.38 \mathrm{~nm}$. These structures were observed in both stretched fibers and single crystals obtained from diluted solutions. New structures based on the establishment of hydrogen bonds along 
two different directions were inferred from diffraction data and energy considerations [5-9]. The molecular conformation of such structures is close to the all trans one since only a slight deviation towards $\sim 150^{\circ}$ (or $-150^{\circ}$ ) for the two $\mathrm{CH}_{2} \mathrm{CH}_{2}-\mathrm{NHCO}$ or $\mathrm{CH}_{2} \mathrm{CH}_{2}$ - $\mathrm{CONH}$ rotation angles of the odd diamine or odd dicarboxylic moeity, respectively, was postulated. This conformation causes amide groups of the odd unit to rotate in opposite senses from the plane defined by methylene carbon atoms. Hydrogen bonds along two directions can be well established when neighboring chains are conveniently shifted, giving rise to a monoclinic unit cell (Figure 1b).

Aliphatic polyamides with a sheet structure usually show a not completely well understood phase transition that occurs on heating. It is named Brill transition and typically consists in a gradual process in which the two strong reflections merge into one on heating, which is indicative of a pseudohexagonal structure [10]. Although a large number of studies have been reported for several nylons [2, 8, 10-17], the phenomenon is not yet fully understood and several explanations have been given: a) thermal expansion anisotropy [2]; b) development of a three-dimensional network of hydrogen bonds [8,10,11], and c) librational motion of methylene groups at high temperature [12]. Simulations of X-ray diffraction patterns and infrared data point to a conformational disorder of methylene groups at high temperature while intermolecular hydrogen bonds between amide groups are kept [18]. The aim of the present work is to gain insight into the structural transitions induced by temperature in odd-even and even-odd polyamides having the new structure with two hydrogen-bond directions. Nylons 65 and 56 were chosen as representative polymers. It should be pointed out that the Brill transition has not yet been studied for any even-odd polyamide and that only data have been reported for some odd-even polyamides (e.g. nylons 11-10 and 11-12 [19]).

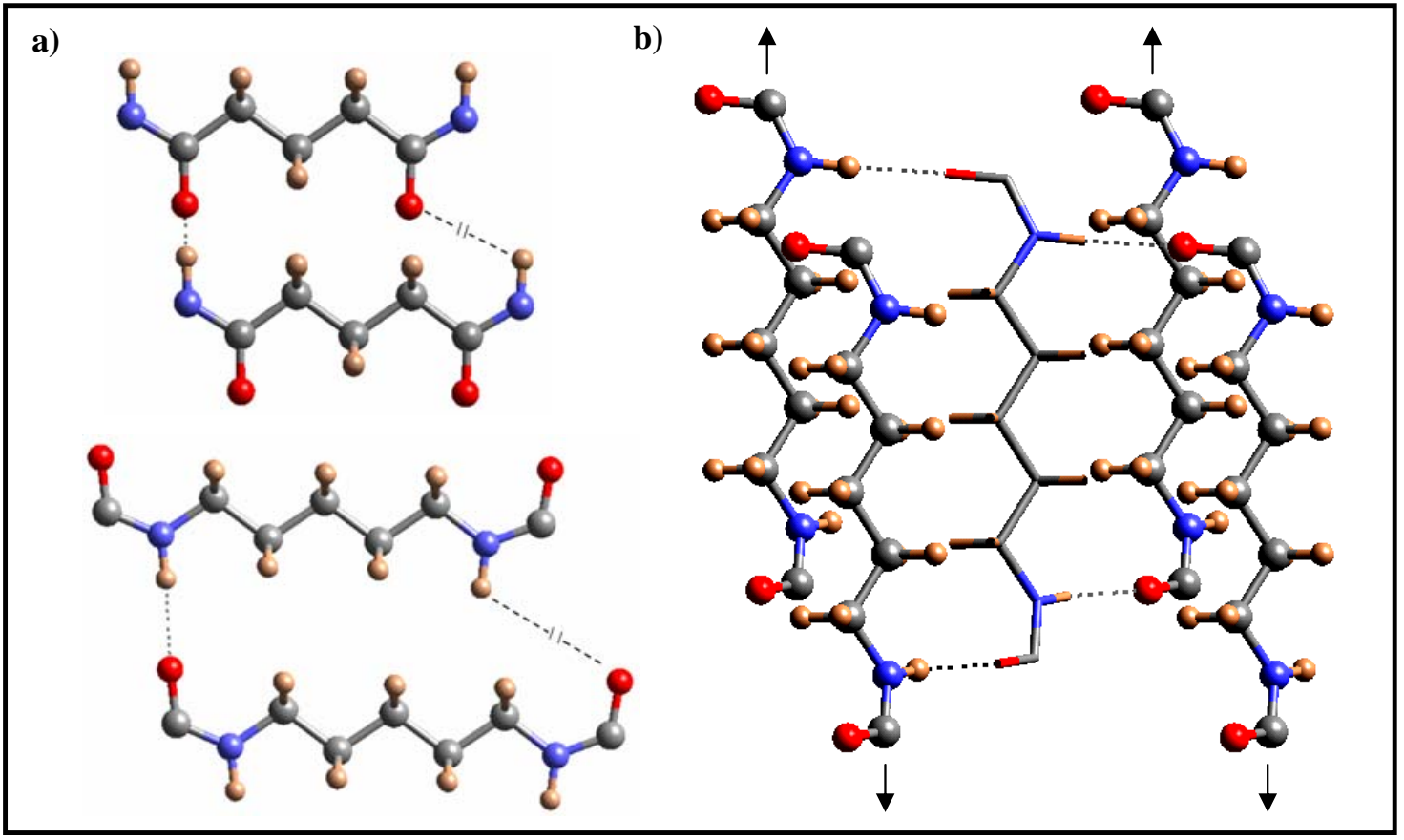

Figure 1. a) Scheme of the unfavorable hydrogen bond geometry between odd diamide units of nylons 65 (up) and 56 (down) when molecular chains have an all trans conformation. b) Scheme of the establishment of hydrogen bonds along two directions when consecutive amide planes of the odd diamide unit of a molecular chain (e.g. nylon 56) slightly rotate in opposite senses from the plane defined by the methylene carbon atoms. External chains (ball and stick representation) should be shifted along the chain axis direction (see arrows) with respect to the central chain (stick representation), thus giving rise to a monoclinic unit cell. Color code: nitrogen, blue; oxygen, red; carbon, gray; hydrogen, brown. 


\section{Experimental procedure}

Nylons 65 and 56 were synthesized by interfacial polycondensation of 1,6-diaminohexane and glutaryl dichloride or 1,5-diaminopentane and adipoyl dichloride, respectively, using toluene as the organic solvent and sodium hydroxide as the proton acceptor, as previously described [8]. Intrinsic viscosities of 0.85 and $0.80 \mathrm{dL} / \mathrm{g}$ were determined in dichloroacetic acid at $25^{\circ} \mathrm{C}$.

$\mathrm{X}$-ray fiber diffraction data were obtained with Ni-filtered $\mathrm{CuK}_{\alpha}$ radiation of wavelength $0.1542 \mathrm{~nm}$ from an Enraf Nonius rotating anode X-ray generator and using a modified Statton camera (W. H. Warhus, Wilmington, DE). Patterns were recorded at different temperatures using a temperaturecontrolled chamber provided by the manufacturer. Oriented fiber samples were obtained by drawing the polymer melt and performing a subsequent annealing under stress at $90^{\circ} \mathrm{C}$.

Time-resolved WAXD experiments were carried out at the CRG beamline (BM16) of the European Synchrotron Radiation Facility of Grenoble. The beam was monochromatized to a wavelength of $0.098 \mathrm{~nm}$ (radiation energy $12.6578 \mathrm{keV}$; spot size $3 \mathrm{~mm}$ (horizontal) x $1 \mathrm{~mm}$ (vertical)). The capillary with the powder sample (as obtained from synthesis) was held on a Linkam hot stage with temperature control within $0.1{ }^{\circ} \mathrm{C}$. WAXD profiles were acquired during heating/cooling experiments in time frames of $12 \mathrm{~s}$. The heating and cooling rates varied between $20-4{ }^{\circ} \mathrm{C} / \mathrm{min}$. The WAXD detector was calibrated with diffractions of a standard of an alumina $\left(\mathrm{Al}_{2} \mathrm{O}_{3}\right)$ sample. The diffraction profiles were normalized to the beam intensity and corrected considering the empty sample background. WAXD peaks were deconvoluted with the PeakFit v4 program by Jandel Scientific Software.

\section{Results and discussion}

The room temperature X-ray diffraction pattern of nylon 65 stretched fibers is mainly characterized by two strong equatorial reflections at 0.432 and $0.375 \mathrm{~nm}$ and several $0 k 0$ reflections defining a $b b^{*}$ angle of $24^{\circ}$ (Figure 2a). All diffraction data can be well indexed according to a monoclinic unit cell ( $a$ $=0.460 \mathrm{~nm}, b$ (chain axis) $=3.095 \mathrm{~nm}, c=0.862 \mathrm{~nm}$, and $\gamma=114^{\circ}$ ) which contains two molecular segments and corresponds to the above structure with two hydrogen bond directions [8].

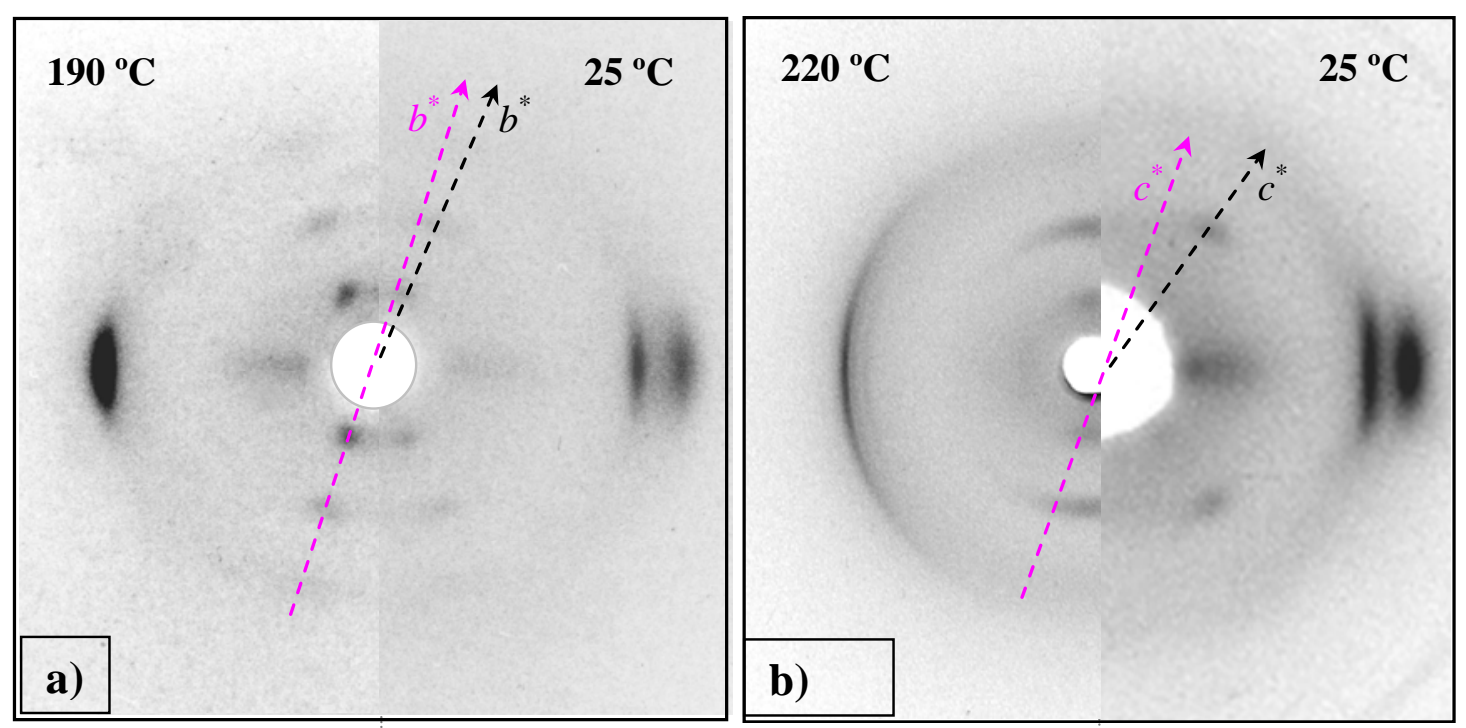

Figure 2. X-ray fiber diffraction patterns of nylons 65 (a) and 56 (b) taken at room temperature and after the Brill transition. Colored and black dashed lines indicate the reciprocal $b^{*}$ (nylon 65) and $c^{*}$ (nylon 56) axes for patterns obtained at the Brill temperature (left side) and at room temperature (right side), respectively.

When the temperature is higher than $190{ }^{\circ} \mathrm{C}$, the pattern shows only one strong equatorial reflection (ca. $0.425 \mathrm{~nm}$ ) and several $0 k 0$ reflections with an off-meridional orientation. Thus, the high 
temperature structure was still characterized by a shift along the chain axis direction between neighbouring chains. In fact, a monoclinic unit cell with $a=0.523 \mathrm{~nm}, b$ (chain axis) $=3.055 \mathrm{~nm}, c=$ $0.850 \mathrm{~nm}$ and $\gamma=110^{\circ}$ can be derived from high temperature diffraction data of nylon 65 . Nevertheless, a pseudohexagonal packing (i.e. the cross-sectioned projection normal to the chain axis, becomes metrically hexagonal) could be suspected by considering the $h 0 l$ reflections only [8].

Figure 3a contains a three-dimensional representation of WAXD profiles of nylon 65 during a heating process performed at $10{ }^{\circ} \mathrm{C} / \mathrm{min}$ from room temperature to fusion for nylon 65. Similar temperature-dependent profiles were observed at the different assayed heating rates. The temperature evolution of diffraction profiles showed three remarkable features: a) The spacings of the two strong equatorial reflections remained practically constant up to a temperature of approximately $70{ }^{\circ} \mathrm{C}$. Then, the intensity of these reflections diminished whereas new ones near 0.426 and $0.400 \mathrm{~nm}$ appeared with increasing intensity. This transition ended at a temperature of approximately $120{ }^{\circ} \mathrm{C}$, indicating a structural change where the parameters of the rectangular chain axis projected unit cell changed from 0.412 and $0.862 \mathrm{~nm}$ (form I) to 0.453 and $0.844 \mathrm{~nm}$ (form II); b) Above $120^{\circ} \mathrm{C}$ the two equatorial reflections $(0.426$ and $0.400 \mathrm{~nm})$ gradually merged into a single peak at $0.425 \mathrm{~nm}$ that was observed just when the temperature reached $190{ }^{\circ} \mathrm{C}$. c) New peaks (e.g. $0.379 \mathrm{~nm}$ ) were detected above $190{ }^{\circ} \mathrm{C}$, suggesting a decrease in crystalline disorder.
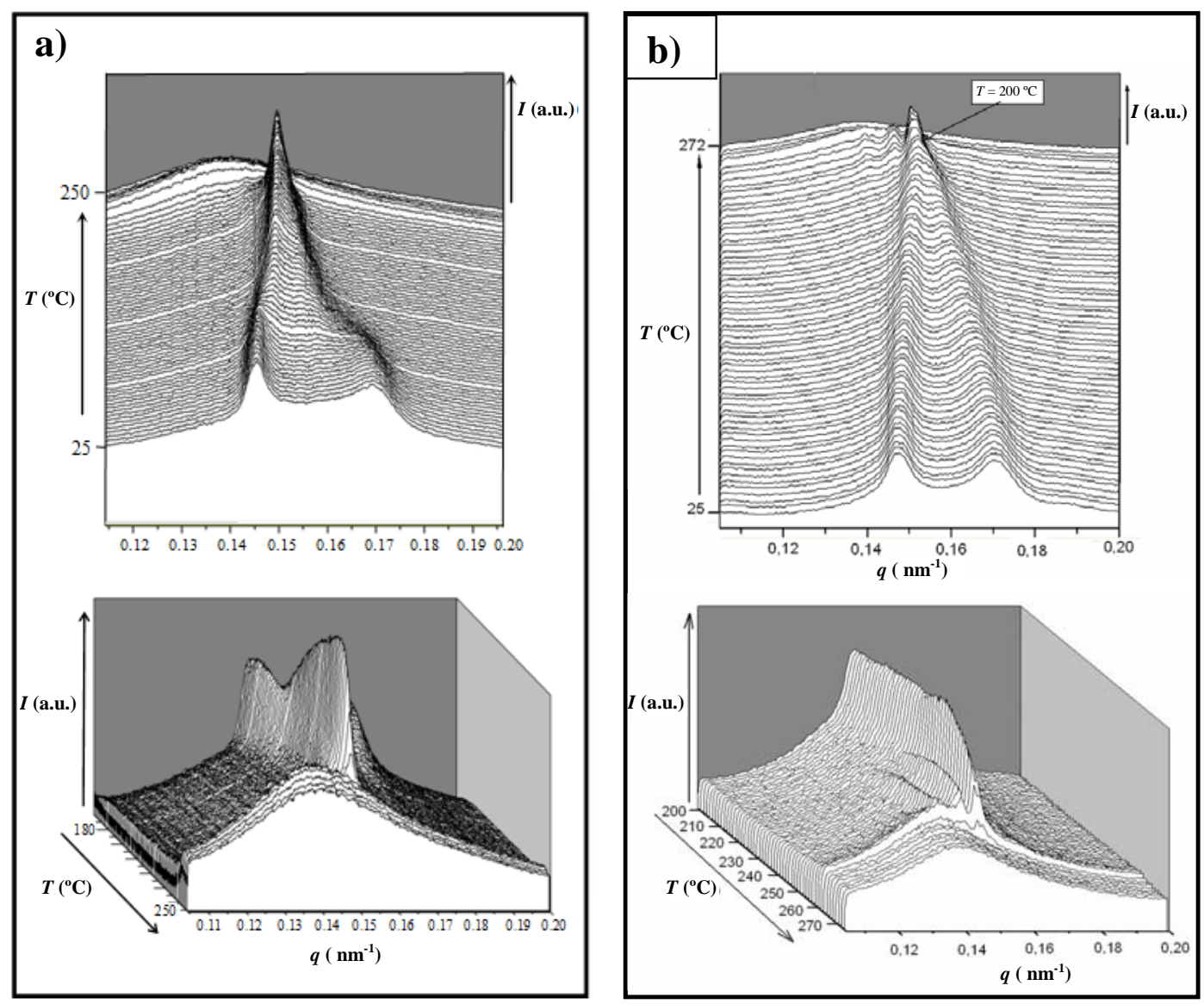

Figure 3. Three-dimensional representations of WAXD profiles of nylons 65 (a) and 56 (b) during heating $\left(10^{\circ} \mathrm{C} / \mathrm{min}\right)$ until fusion from room temperature (up) and from $\sim 70{ }^{\circ} \mathrm{C}$ before fusion (down). 
The two observed transitions may involve only slight changes in the torsional angles vicinal to amide groups or even an increase in the mobility of polymethylene segments, which could lead to a hexagonal packing and a monoclinic structure without disrupting the initial hydrogen-bonding scheme. In this sense, fiber patterns with off-meridional $0 k 0$ reflections are essential to support the finding that the structure observed at high temperature $\left(>190^{\circ} \mathrm{C}\right)$ is different from the pseudohexagonal structure attained with conventional even-even polyamides after the Brill transition.

Figure 4a shows the WAXD profiles of nylon 65 obtained during a cooling run $\left(10^{\circ} \mathrm{C} / \mathrm{min}\right)$ from the melt state. Between 190 and $165^{\circ} \mathrm{C}$ crystallization occurred and a narrow peak appeared around 0.420 $\mathrm{nm}$ with increasing intensity. This peak started to split when the temperature was close to $130{ }^{\circ} \mathrm{C}$, which could be considered the Brill transition temperature on cooling. A hysteresis effect was consequently observed (i.e. transitions took place at $190{ }^{\circ} \mathrm{C}$ on heating and at $130{ }^{\circ} \mathrm{C}$ on cooling), as usually reported for conventional polyamides. Cooling profiles also show that a new split occurred around $100{ }^{\circ} \mathrm{C}$, which can be interpreted as a transition from form II to form I. This structural change was not complete even if the cooling rate was decreased to $4{ }^{\circ} \mathrm{C} / \mathrm{min}$. Thus, deconvolution of the diffraction profile obtained at room temperature (Figure 5a) revealed two amorphous halos and four crystalline peaks appearing at 0.430 and $0.379 \mathrm{~nm}$ (form I) and 0.422 and $0.403 \mathrm{~nm}$ (form II). A complete conversion to form I was only attained when fibers were subsequently annealed under stress at $80{ }^{\circ} \mathrm{C}$ or when the sample was recrystallized from diluted glycerine solutions.
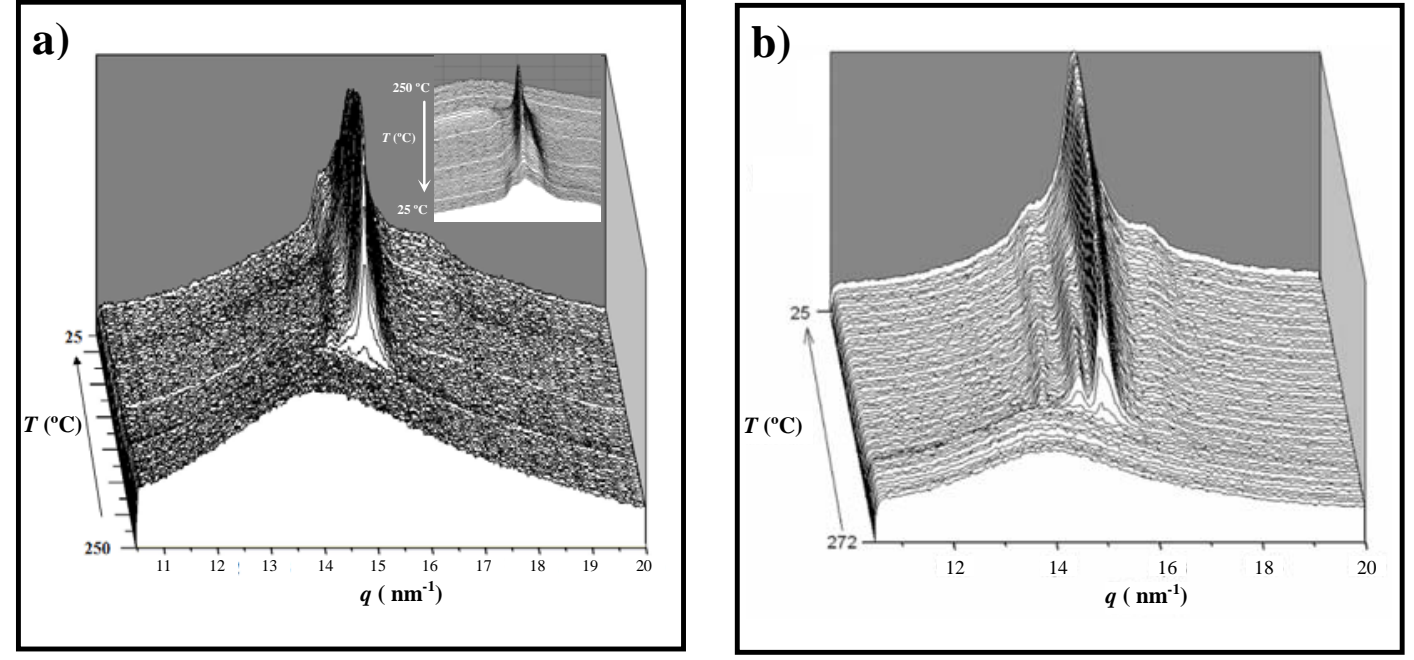

Figure 4. a) Three-dimensional representation of WAXD profiles of nylons 65 (a) and 56 (b) during cooling $\left(10^{\circ} \mathrm{C} / \mathrm{min}\right)$ from the melt to room temperature.

The X-ray fiber diffraction pattern of nylon 56 is also characterized by two strong equatorial reflections at spacings of 0.432 and $0.375 \mathrm{~nm}$ and off-meridional $00 \mathrm{l}$ reflections (Figure 2b). These define a $c c^{*}$ angle close to $36^{\circ}$, a monoclinic unit cell containing two chain segments and dimensions of $a=0.512 \mathrm{~nm}, b=0.864 \mathrm{~nm}, c$ (chain axis) $=3.133 \mathrm{~nm}$, and $\beta=125.7^{\circ}$ [5] being inferred. At $220^{\circ} \mathrm{C}$ the diffraction pattern shows a dramatic change (Figure 2b). As can be seen, only a single strong equatorial reflection appeared $(0.423 \mathrm{~nm})$ and the $00 \mathrm{l}$ reflections became close to the meridian. It is remarkable that new weak reflections with a close equatorial orientation (e.g. 0.454 and $0.436 \mathrm{~nm}$ ) also appeared. These could be indexed assuming a monoclinic unit cell with $a=0.551 \mathrm{~nm}, b=0.846$ $\mathrm{nm}, c$ (chain axis) $=3.133 \mathrm{~nm}$, and $\beta=112.6^{\circ}$. Note that the arched reflections caused by disorientation and the reduced $c c^{*}$ angle may account for the practically meridional appearance of the 00l reflections. 
Changes in the three-dimensional WAXD profiles during heating at $10{ }^{\circ} \mathrm{C} / \mathrm{min}$ are shown in Figure $3 \mathrm{~b}$. It is clear that the two equatorial reflections gradually merged into a single peak at $0.423 \mathrm{~nm}$ according to a Brill transition that was completed when the temperature reached $200{ }^{\circ} \mathrm{C}$. In this case, no intermediate crystalline structure was favored during heating. The dynamic analysis also revealed that between 230 and $235{ }^{\circ} \mathrm{C}$ the intensity of the strong reflection slightly increased and the peak became narrower. In this temperature range new reflections also appeared and increased in intensity before the start of a melting process that finished at $255^{\circ} \mathrm{C}$. It seems that at a high temperature $(\sim 230$ $\left.{ }^{\circ} \mathrm{C}\right)$ a new monoclinic structure developed. Its diffraction pattern shows narrow peaks, which suggest an improved arrangement of the amide group. Note that the motion of methylene groups may increase with temperature without affecting the diffraction pattern since the observed reflections are mainly a consequence of the orderly disposition of the constitutive amide groups.

Figure 4b contains the WAXD profiles on nylon 56 acquired during a cooling run performed at 10 ${ }^{\circ} \mathrm{C} / \mathrm{min}$ from the melt state. Two interesting distinctive features not observed in conventional nylons are shown:

a) The polymer mainly crystallized in the high temperature structure, which remained unchanged throughout the cooling process. Thus, the Brill transition was not reversed and only the disappearance of the small and narrow peaks of the high temperature profiles was detected (e.g. the $0.436 \mathrm{~nm}$ reflection gradually shifted to a lower spacing until merging with the predominant peak at $0.423 \mathrm{~nm}$ ).

b) Reflections characteristic of the low temperature monoclinic structure practically started to appear at the beginning of the crystallization process. However, overlapping with some weak reflection of the high temperature structure made it difficult to discern the precise instant at which these new reflections appeared. In any case, it is clear that these reflections did not come from a split of the strong peak at $0.423 \mathrm{~nm}$ although their intensity slightly increased during cooling. The spacings of the considered reflections slightly decreased during cooling as a consequence of the cell contraction being observed at 0.440 and $0.383 \mathrm{~nm}$ when room temperature was reached.

Deconvolution of the diffraction profile obtained at room temperature (Figure 5b) clearly revealed two amorphous halos and four crystalline peaks that demonstrate the coexistence of the low temperature monoclinic structure (i.e. 0.440 and $0.383 \mathrm{~nm}$ reflections) and the structure characterized by the pseudohexagonal packing favored at high temperature (i.e. 0.419 and $0.405 \mathrm{~nm}$ reflections). As in the case of nylon 65, a complete conversion to the low temperature structure could only be attained when fibers were subsequently annealed under stress at $80^{\circ} \mathrm{C}$ or when the sample was recrystallized from a diluted formic acid-ethanol (1:4) solution [5].

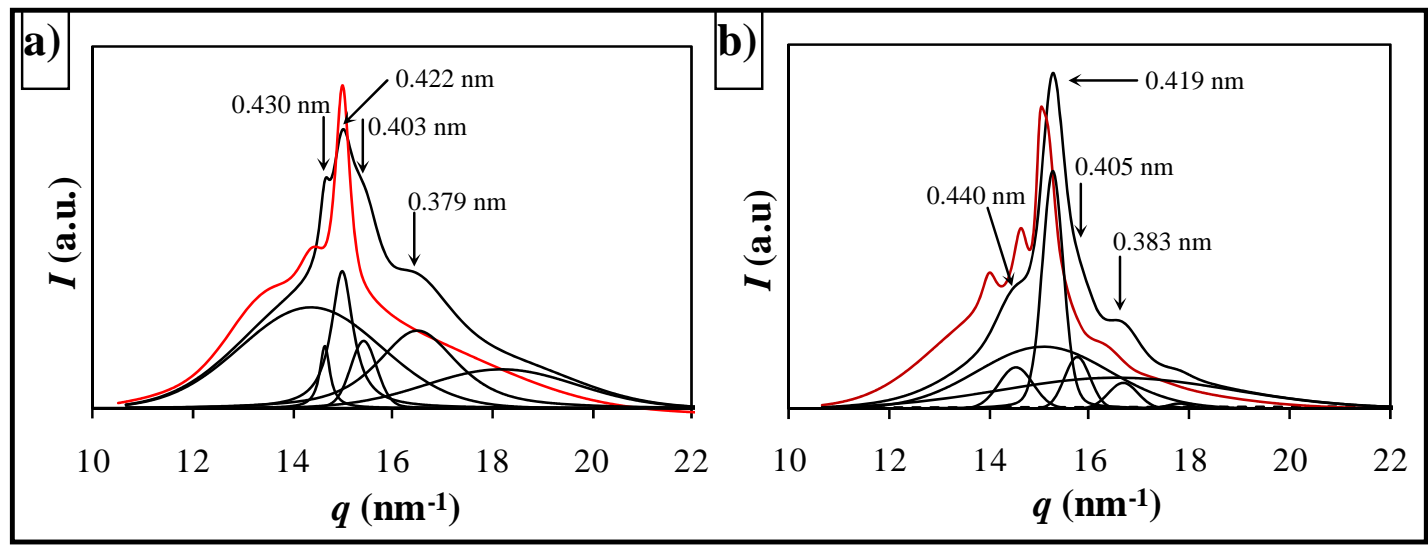

Figure 5. One-dimensional WAXD profile (black) for nylons 65 (a) and 56 (b) taken at room temperature after a cooling run $\left(10^{\circ} \mathrm{C} / \mathrm{min}\right)$ from the melt state. Spacings of main reflections are indicated together with the deconvoluted peaks. For the sake of completeness profiles taken just before melting are also shown (red curves). 


\section{Conclusions}

Nylons 65 and 56 showed a Brill transition on heating that rendered monoclinic structures clearly different from the pseudohexagonal structures usually postulated for conventional polyamides. Although both polymers had a similar structure at room temperature when obtained from solution crystallization, significant differences were found between the two studied polyamides during the heating and cooling processes. This structure was characterized by the establishment of intermolecular hydrogen-bonding interactions along two directions and a fiber diffraction pattern with two strong equatorial reflections close to 0.432 and $0.375 \mathrm{~nm}$.

On heating, nylon 65 first showed a transition towards a less compact structure (form II) characterized by the presence of two close equatorial reflections $(0.426$ and $0.400 \mathrm{~nm})$ and subsequently underwent a second transition where the two indicated reflections progressively merged into a single one. In contrast, nylon 56 only showed a transition towards a pseudohexagonal packing although an additional rearrangement of amide groups was detected at some degrees before fusion. Structural changes may occur on heating for both nylons without disrupting the hydrogen bonding scheme and involving only a small change in the angle between consecutive amide groups of the odd unit.

Nylons 65 and 56 crystallized from the melt according to the Brill high temperature structure. In the case of nylon 65, the structure first reverted to form II on cooling, showing a hysteresis effect, and then this form II underwent a partial transition to the low temperature structure. On the contrary, nylon 56 did not show a Brill transition during the cooling process although a progressive, minor crystallization into the low temperature structure also took place.

\section{Acknowledgments}

This research has been supported by CICYT and FEDER grants (MAT 2006-02406 and MAT200911503). We want to express our gratitude to Drs. François Fauth and Ana Labrador of the CRG BM16 beamline staff of CELLS (Consortium for the Exploitation of the Synchrotron Light Laboratory).

\section{References}

[1] Xenopoulos A and Clark E S 1995 Nylon Plastics Handbook, ed M I Kohan (Munich, Vienna and New York: Hanser Publishers) chapter 5 pp 108-135.

[2] Holmes D E, Bunn C W and Smith D 1955 J Polym Sci Part A, General Papers 17159

[3] Bunn C W and Garner E V 1947 Proc R Soc London Ser A 18939

[4] Kinoshita Y 1959 Makromol Chem 331

[5] Puiggalí J, Franco L, Alemán C and Subirana J A 1998 Macromolecules 31, 8540

[6] Franco L, Subirana J A and J. Puiggalí. 1998 Macromolecules 31, 3912

[7] Villaseñor P, Franco L, Subirana J A and Puiggali J 1999 J Polym Sci Part B, Polym Phys Ed 37, 2383

[8] Navarro E, Franco L, Subirana J A and Puiggalí J 1995 Macromolecules 28, 8742

[9] Franco L, Cooper S J, Atkins A D T, Hill M and Jones N A 1998 Macromolecules 36, 1153

[10] Brill R 1956 Makromol Chem 18, 294

[11] Schmidt G F and Stuart H A 1958 Z Naturforsch 13A, 222

[12] Hirschinger J, Miura H, Gardner K H and English A D 1990 Macromolecules 23, 2153

[13] Ramesh C, Keller A and Eltink S J E A. 1994 Polymer 35, 2483

[14] Hill M J and Atkins E D T 1995 Macromolecules 28, 2642

[15] Murthy N S, Wang Z and Hsiao B S 1999 Macromolecules 32, 5594

[16] Ramesh C, and Gowd E B 1999 Macromolecules 32, 3721

[17] Feldman A Y, Wachtel E, Vaughan G B M, Weinberg A and Marom G. 2006 Macromolecules 39, 4455

[18] Tashiro, K and Yoshioka, Y 2004 Polymer 45, 6349

[19] Cui X, Yan D 2005 Eur Polym J 41, 863. 